\title{
EL DISCURSO DE LA UNIÓN EUROPEA SOBRE LA SOCIEDAD DEL CONOCIMIENTO
}

\author{
Amparo Serrano \\ European Trade Union Institute, Bruselas
}

Eduardo Crespo

Universidad Complutense

\begin{abstract}
RESUMEN
El discurso acerca de la sociedad del conocimiento y de la información ha sido integrado y popularizado por las instituciones europeas, y ha sido vinculado al proyecto social europeo. Este discurso implica, entre otras cuestiones, una redefinición de la cohesión social, que es caracterizada por un contexto de crisis. Esta crisis se extendería en torno a tres ejes: una crisis de regulación del mercado de trabajo, una crisis del desempleo y una crisis de la ciudadanía. El nuevo contrato social planteado en el discurso de las instituciones de la Unión Europea se articula básicamente en torno a estos tres ejes: frente a la crisis de la regulación del trabajo, el discurso de la flexibilidad del trabajo; frente a la crisis del desempleo, el discurso de la empleabilidad, y frente al modelo de intervención social basado en los derechos ciudadanos, el discurso de la activación y la responsabilidad personal.
\end{abstract}

\section{INTRODUCCIÓN}

Los términos «sociedad basada en el conocimiento y la información», y el más genérico aún de "nueva economía», constituyen ya parte del acervo común en las explicaciones económicas aportadas por los medios de comunicación y los foros de discusión política. Según este tipo de discurso, los sistemas de producción industrial, basados en un uso intensivo de energía, estarían siendo sustituidos por sistemas de producción intensivos en información, transformándose, de este modo, las nuevas tecnologías de la información y 
comunicación en factor de producción, de competitividad y de crecimiento económico. Estas tecnologías permitirían la reducción de los costes de producción, un aumento de la velocidad en el procesamiento de datos y constituirían un potencial de innovación productiva, implicando la liberación de límites espaciales y temporales, así como otro tipo de barreras (Tyson, 2000).

Las instituciones europeas han hecho suyo este tipo de terminología y lo vienen aplicando al análisis de la situación económica y al diseño de propuestas y políticas sociales. El principal eje de este discurso se dirige a la necesidad de dotar a Europa de una infraestructura tecnológica adecuada a esta nueva fase económica, así como de la formación adecuada a los trabajadores para aplicar y utilizar tales tecnologías.

No es nuestra pretensión analizar lo adecuado de las explicaciones económicas que se están aportando, sino señalar que el discurso de la Unión Europea sobre la «nueva economía» o la "sociedad basada en el conocimiento» va más allá de los recientes desarrollos tecnológicos, vinculados a la informática y a las comunicaciones. Este discurso plantea los efectos de las nuevas tecnologías en la organización del trabajo, en la noción de empleo y en la demanda de cualificaciones. En este sentido, este discurso no solamente propone una teoría acerca de las recientes transformaciones económicas, sino que implica un proceso de redefinición social e ideológica, dirigido a crear un nuevo marco de legitimación de la actividad económica. Establece una nueva "normalidad» y supone una rearticulación del sentido del trabajo, del tiempo y de las relaciones personales.

El discurso acerca de la sociedad del conocimiento y de la información ha sido integrado y popularizado por las instituciones europeas, $y$ ha sido vinculado al "proyecto social europeo». Estos últimos tres años, las instituciones europeas han sido particularmente activas en cuanto a la producción y extensión de documentos acerca de la cohesión social y de la dimensión social del proyecto europeo. Estos discursos, que en su origen tenían, más bien, un cariz de defensa de la ciudadanía social, como un necesario complemento de la dimensión económica y una preocupación por el frágil y descuidado equilibrio social (véase, por ejemplo, el llamado proceso de Luxemburgo), cada vez desarrollan más un tono tecnológico y economicista, dirigido a la construcción de una respuesta frente a los nuevos desafíos económicos. En este sentido, lo que se plantea no es tanto un complemento a una insuficiente unión europea en el terreno social, sino más bien una especie de necesidad ante una evolución económica que se impone como tal. Este discurso ha sido particularmente fecundo tras la presidencia finlandesa y, en especial, de la presidencia portuguesa. Veamos, en primer lugar, algunos aspectos de la estructura argumentativa que caracteriza a este discurso, para pasar, a continuación, a analizar los ejes en torno a los cuales se articula lo que consideramos una visión individualizante de la solidaridad y de la "cuestión social». 


\section{ESTRUCTURA ARGUMENTATIVA DEL DISCURSO DE LA SOCIEDAD DEL CONOCIMIENTO}

Las instituciones europeas han sido particularmente fecundas los últimos años en la producción de discursos acerca de la sociedad del conocimiento ${ }^{1}$. La estructura general de este tipo de discurso podríamos caracterizarla, en primer lugar, por la naturalización histórica; en segundo lugar, por la continua referencia a una dicotomía entre la economía y sociedad americana y la europea, y, finalmente, por plantear una teoría acerca de un nuevo modo de articular el vínculo social.

\section{Naturalización histórica}

Con la metáfora de la economía del conocimiento se plantea de modo implícito una teoría acerca de la dirección del cambio social. Ante un cambio tecnológico, económico y social, que se presenta como evidencia, se considera como algo obvio el carácter «necesario» de la modernización social e institucional (reformas estructurales, extensión de la formación a las nuevas tecnologías, etc.). Esta modernización aparece, por tanto, como la respuesta "natural» a la transformación y globalización económica². Esta naturalización del proceso

${ }^{1}$ Por ejemplo, European Union, European Commission, Information society technologies. Challenges and opportunities, Luxembourg: EUR-OP; European Union, European Commission (1999), eEurope. An information society for all. Communication on a Commission initiative for the special European Council of Lisbon, 23 and 24 March 2000, COM (1999), Luxembourg: EUR-OP; European Union, European Commission (1999), Strategies for jobs in the Information Society, Luxembourg: EUR-OP, 2000; European Union, European Commission (2000), eEurope. An information society for all. Progress report. For the special European Council of employment reforms and social cohesion. Towards a Europe based on innovation and knowledge, Lisbon, 23 and 24 March 2000, COM (2000), Luxembourg: EUR-OP, 2000; European Union, European Commission (2000), eEurope 2002, An information society for all, Draft action plan prepared by the European Council in Feira 19-20 June 2000, COM (2000), Luxembourg: EUR-OP, 2000; European Union, European Commission (2000), Proceedings of the DLM-Forum on electronic records. European citizens and electronic information: the memory of the information society, Brussels, 18-19 October 1999, Luxembourg: EUR-OP, 2000; European Union, European Commission (2000), IST 2000, Realising an information society for all, Luxembourg: EUR-OP, 2000. 2272 Council RESEARCH. 15.06.2000. Press: 210- Nr: 9411/00; Council of the European Union (2000) Portuguese presidency of the European Union - Programme. 18.01.2000. Nr: 1080/1/00. Lisbon European Council: Presidency conclusions. 24-03-2000. Nr: 100/1/00. Document submitted to the Lisbon European Council: Contribution by the Labour and Social Affairs Council. 24.03.2000. Nr: 6966/00. Open Council debates during the Portuguese presidency, 20.01.2000. Santa Maria Da Feira European Council: Presidency conclusions. 10-06-2000. Nr: 200/1/00. Estos documentos del Consejo Europeo están disponibles en la web del Consejo.

2 "The European Union is confronted with a quantum shift resulting from globalisation and he challenges of a new knowledge-driven economy. These changes are affecting every aspect of people's lives and require a radical transformation of the European economy. The Union must shape these changes in a manner consistent with its values and concepts of society...». Lisbon Earopean Council. Presidency conclusions, 24.03.2000, Nr. 100/1/00, p. 1. 
hace que éste aparezca inscrito en el orden de la naturaleza, fuera de la voluntad de las personas. La expresión «sociedad del conocimiento» designa así no tanto una decisión política cuanto la gestión técnica del cambio. Se realiza así una reconstrucción histórica de las evoluciones contemporáneas que, mediante la naturalización de estos desarrollos, hacen de la modernización una necesidad.

Estas nuevas condiciones económicas han sido definidas de formas muy diversas (sociedad postindustrial, sociedad postfordista, nueva economía, etc.). Sin embargo, las instituciones europeas van a hacer de la información el articulador central de estos nuevos cambios, usando, con frecuencia, la expresión de sociedad de la información y del conocimiento. La comunicación y la información van a ser así el factor de producción determinante para la competitividad industrial. Si esta economía tiene, para algunos, poco de nueva (Evans, 2000; Visco, 2000), lo que sí es novedoso es la popularidad de este discurso acerca de la sociedad basada en el conocimiento y el centramiento de este discurso en el impacto de los aspectos tecnológicos como portadores de un nuevo modelo de hacer sociedad.

\section{El modelo americano como referente del modelo europeo}

Otro carácter importante de este discurso institucional es la dependencia simbólica frente al modelo americano, que hace que tenga a éste como interlocutor y del cual proclama a su vez su distancia ${ }^{3}$. El objetivo último del proyecto europeo sería aventajar en la posición competitiva a la economía americana. Del job miracle american se subrayan los aspectos ligados al liderazgo en la difusión de tecnologías de información, pero también los diferenciados modos de regulación social que actúan como condición necesaria de este crecimiento. ¿En qué consistiría entonces la especificidad del modelo europeo? Observamos el recurso a otro concepto también de moda en el discurso de la Comisión: «la cohesión social». Se trataría de construir una sociedad basada en la información y en el conocimiento para todos (inclusive society). La lucha contra la

3 «Europe has major weaknesses and is well behind the United States in the use of the new information and communication technologies». Communication from the Commission, e-Learning Designing tomorrow's education. Brussels, 24.5.2000, COM (2000), p. 5.

"The Union has today set itself a new strategic goal for the next decade: to became the most competitive and dynamic knowledge-based economy in the world, capable of sustainable economic growth with more and better jobs and greater social cohesion. Achieving this goal requires an overall strategy aimed at: preparing the transition to a knowledge based economy and society by better policies for the information society and $\mathrm{R} \& \mathrm{D}$, as well as by stepping up the process of structural reform for competitiveness and innovation and by completing the internal market; - modernising the European social model, investing in people and combating social exclusion, - sustaining the healthy economic outlook and favourable growth prospects by applying an appropriate macro-economic policy mix...». Lisbon European Council. Presidency conclusions, 24.03.2000, Nr. 100/1/00, p. 2. 
exclusión social se basaría en la difusión democrática y universal de las tecnologías de información y de las competencias para su utilización, así como su extensión a todas las capas sociales ${ }^{4}$. El progreso tecnológico iría, pues, seguido de un progreso social. En este sentido, parecería que dotándose a los trabajadores de las nuevas herramientas tecnológicas, y de los conocimientos suficientes para utilizarlas, el proceso de inserción en el mercado de trabajo seguiría automáticamente. El problema de exclusión de una parte de la población radicaría en la falta de estas competencias, con lo cual la democratización de éstas, y su extensión a todas las capas sociales, permitiría una sociedad integradora por excelencia.

\section{Rearticulación de las responsabilidades sociales}

Si bien el discurso se centra en la necesaria dotación de habilidades técnicas al sujeto para la adaptación a las nuevas condiciones de producción, también se pone el acento en el papel que la organización del trabajo y de la sociedad tiene en este proceso. De ahí que no pueda limitarse el concepto de conocimiento a los sistemas de información. Las nuevas tecnologías de la información constituyen un apoyo del proceso ya que pueden contribuir a aumentar la estandarización y difusión de conocimientos, pero no lo generan en sí mismo. Más que la formación de los trabajadores, es la organización del trabajo la que jugará un papel central en que estos conocimientos se transformen en innovaciones y en respuestas creativas a estas nuevas condiciones. En este sentido, el papel de los conocimientos informales y «tácitos» (Valenduc, 2000), fruto de la experiencia en el trabajo, tiene tanta o más importancia que los conocimientos formalizados adquiridos a través de la formación.

Sin embargo, este discurso no acaba aquí. La infraestructura y la formación son condiciones necesarias, pero no suficientes, para esta adaptación de la

${ }^{4}$ "The implication of the Lisbon conclusions is that $e$ Europe should extend the focus of its $e$ Participation action. The benefits of the information society must be accessible, ... to those outside of the labour market and the educational system...». e Europe 2002 An information society for all, 24.5.2000, COM (2000), p. 17.

«It calls for attention to education, and training policies, in particular with the view that life long learning is becoming vital if people are to be empowered to act as full members of the knowledge and information society». Communication from the Commission. Building an inclusive Europe, 1.3.2000, COM (2000), p. 6.

"At the heart of many of the employment challenges identified in this Communication, ... lies questions of human resources and capabilities. Ensuring a wide distribution and high level of skills and knowledge is central to the development of a dynamic and inclusive knowledge economic...». Communication from the Commission, Brussels, 1.3.2000, COM (2000), p. 21.

5 "The challenge is wider than just meeting the demand for information technology professionals. Digital literacy is an essential element of the adaptability of all citizens. ... the third challenge lies in the modernisation of work organisation. Greater flexibility bring the technological benefits of variable time and place of work to people in work...». European Commission. $e$ Europe 2002. An information society for all, Brussels, 24.5.2000, COM (2000), p. 15. 
sociedad europea a los nuevos desafíos. Son así necesarias no sólo nuevas formas de ejercicio del trabajo que permitan la adquisición y desarrollo de competencias, sino, sobre todo, nuevas comprensiones de lo social y, en este sentido, formas renovadas de entender la relación entre derechos y responsabilidades. Este discurso plantea la necesaria «modernización» o adaptación de los recursos humanos, así como de las instituciones, a estas nuevas condiciones. Se trata de una naturalización de la necesidad de reforma de las instituciones en las que se ha basado el modelo social de las sociedades europeas industriales (Estado social, regulaciones del mercado de trabajo, institucionalización del trabajo, etc.). Las instituciones en las que se ha basado este modelo son así acusadas de la mayor de las perversiones posibles, generar una "euroesclerosis», esto es, estar en el origen de la crisis del trabajo.

El discurso de la Comisión acerca de la knowledge based society coincide con un proceso de construcción de una identidad europea, y particularmente de un proceso de búsqueda de los valores en los que articular esta identidad europea $^{6}$. En esta búsqueda se plantea como una evidencia la inadaptación del modelo social y económico europeo a las nuevas condiciones económicas y sociales. Las reglas del modelo industrial aparecen inadecuadas a las nuevas condiciones económicas ${ }^{7}$. Esta sociedad demandaría así nuevas exigencias normativas, nuevas competencias (técnicas, metodológicas y morales) por parte del trabajador, pero también nuevas estructuras reguladoras de las instituciones del trabajo (más procedimentales y flexibles que sustantivas) ${ }^{8}$.

6 «Europe must find its own way of constructing an innovation and Knowledge based society and economy. The European way needs to open up opportunities for accessing knowledge, value cultural diversity at its true worth and use this transition in order better to forge a specific European identity and to identify citizens more closely with a European project which they themselves will define». Document for the presidency, Employment, Economic reforms and Social cohesion- towards a Europe based on innovation and knowledge. Council of the European Union, 12.1.2000, 5256/00, p. 6.

«Digital technologies are transforming the old rules that governed a predominantly industrial society to a new set of rules-those of the Information society... The term new economy describes the transformation of economic activities that is taking place as digital technologies make accessing, processing and storage of information increasingly cheaper and easier» (e Europe An information society for all. Progress Report. For the special European council on Employment, Economic reforms and Social Cohesion- Towards a Europe based on innovation and knowledge. Lisbon, 23 and 24 March, 8.3.2000, COM (2000), p. 1, annex 2.

8 «Despite a number of undeniable successes, Europe is lagging behind in this transition to the innovation and knowledge-based economy. The delay is apparent in the production and dissemination of much information technology but also in adaptation of social institutions and relations the new potential opened up by such technology. While this failure to adapt to the new paradigm continues, there will be a shortfall in economic growth and an increased risk of unemployment and social exclusion. We need to increase the pace of technological change but also of institutional reform... An economic and social strategy to renovate the basis for growth in Europe must combine macro-economic policies, economic reform and structural policies, active employment policies and the modernisation of social protection (p. 5). Nowadays it is becoming clear that the problem is not only about information, but about knowledge and innovation, and not only about technological change, but also about economic and social change (p. 6). «Docu- 
Esta construcción del así llamado «modelo europeo» es el resultado de una importante crisis de identidad del proyecto europeo?. Parecería como si la evaluación de la eficacia o no de la estrategia europea se viera afectada por la evidencia de la ausencia de un modelo europeo claramente definido. Este proceso explica la importancia del discurso de la presidencia portuguesa acerca de la así llamada «sociedad de la información» o «sociedad del conocimiento».

\section{PRINCIPIOS NUCLEARES DE ESTE MODELO SOCIAL EUROPEO}

El discurso de la sociedad basada en el conocimiento es, por tanto, también un discurso acerca de la cohesión social, discurso éste construido bajo un fondo de crisis. Esta crisis se extendería en torno a tres ejes, una crisis de regulación del mercado de trabajo, una crisis del desempleo y una crisis de la ciudadanía. El nuevo contrato social planteado por las instituciones se articula, por tanto, en torno a estos tres ejes: A) frente a la crisis de la regulación y normalización fordista del trabajo, la «invención» ${ }^{10}$ de la flexibilidad postindustrial; B) frente a la crisis del desempleo (de la solidaridad y socialización de la responsabilidad), la «invención» del concepto de empleabilidad, y C) frente al modelo de intervención social basado en la ciudadanía, la «invención» de la activación.

Estas tres dimensiones están actuando como los ejes en torno a los cuales se gestiona el cambio, que permitirían la adaptación a las nuevas presiones del mercado y la evolución de una economía basada en el conocimiento. Esto implicaría la adaptación de las instituciones del Estado de bienestar, así como el fomento de competencias y capacidades a diversos niveles (competencias personales —empleabilidad—, de ciudadanía —activación — y nuevos modos de regulación social —flexibilidad—).

ment for the presidency, Employment, Economic reforms and Social cohesion- towards a Europe based on innovation and knowledge». Council of the European Union, 12.1.2000, 5256/00.

9 "The European Union, which for decades has structured a political, economic and social project based on a set of values which today defines its own particular civilisational model, has been assuming special responsibility for creating conditions enabling the peoples of this continent to live in societies characterised by freedom, security and progress... the reunification of Europe demands today not only a deepening of the European Union integration project but also that it be shared with all States that respect the essential values on which such a project is based». Portuguese presidency of the European Union. Programme. SN 1080/1/00 rev1. 13.1.2000, p. 2.

${ }^{10}$ En el sentido que R. Salais, Baverez y Reynaud (1986) plantean en su libro L'invention du chômage. 


\section{NuEVAS REGULACIONES DEL TRABAJO: DE LA NORMALIZACIÓN FORDISTA A LA FLEXIBILIDAD POSTFORDISTA}

\section{Cambios económicos y demanda de nuevas regulaciones}

Según este discurso dominante, el nuevo paradigma productivo sometería a las empresas a una competición constante por la calidad, la variedad, la innovación y el tiempo. Este contexto demanda formas renovadas de regulación de la mano de obra ajustadas a condiciones de imprecisión y complejización social. La inversión en capital humano aparece como una importante vía para la competencia y el crecimiento económico, lo que implica una regulación de las tareas que fomente la implicación y motivación de los trabajadores (Wood, 2000). El factor humano y los aspectos cognitivos y actitudinales (iniciativa, responsabilidad, motivación, implicación y participación directa de la fuerza de trabajo) adquieren así un papel central frente al trabajo regulado y pasivo de la era taylorista ${ }^{11}$. La producción industrial clásica, consistente no sólo en un sistema de producción de normas de organización de la producción (estandarización de la producción, etc.), sino también en una forma de clasificación de los puestos de trabajo, resulta inadecuada. Se trata fundamentalmente de un sistema de regulación en el que el trabajador pierde en gran medida su autonomía. Las nuevas condiciones productivas demandan más la iniciativa que la sumisión, más la competencia que la cualificación (Maroy, 1997). Tres tipos de competencias son particularmente valoradas: competencias comunicativas, competencias cognitivas para hacer frente a situaciones urgentes y competencias vinculadas a la iniciativa y a la creatividad. Se establece así un nuevo compromiso entre un modo de organización más flexible por parte de la dirección y una demanda de lealtad e implicación con la empresa por parte de los trabajadores $^{12}$.

Las tecnologías de la información han favorecido en algunas empresas formas de organización flexibles, poco jerarquizadas y formalizadas (Askenazy, 2000), que permitan el aprendizaje y la transmisión de la información a todos los niveles y una organización del trabajo en redes, a fin de facilitar el intercambio de comunicación entre individuos y organizaciones. Por ello, la sociedad del conocimiento, más allá de una mera actividad económica, se trata de una nueva forma de organización de lo social ${ }^{13}$. Sin embargo, en la mayor

${ }_{11}$ Tal y como plantea De Coster (1998), la esencia del taylorismo no sería tanto la parcelación de las tareas como la distinción radical entre la concepción del trabajo, confiada a un departamento de expertos, y la ejecución (atributo exclusivo de los obreros).

12 Este proceso estaba en marcha desde hace algunos años, pero, tal y como ponen de manifiesto diversos estudios (Askenazy, 2000; Rubenson y Schuetze, 2000), la «nueva economía» ha acelerado este proceso.

${ }_{13}$ Potential also requires stock to be taken of the European social model, which is one of the strong suits of the European project. But there are two prerequisites for its continuation in the context of globalisation: the renovation of its economic base, building new competitive factors 
parte de las organizaciones, la integración de nuevas tecnologías no ha ido acompañada de reales cambios organizacionales (Askenazy, 2000; Brödner, 2000; G. Duval y H. Jacot, 2000).

\section{Flexibilidad y soberanía en el tiempo de trabajo}

Las nuevas tecnologías de la información y de la comunicación han posibilitado, en algunas ocasiones, organizaciones más flexibles que permiten al trabajador asumir más responsabilidades, más autonomía y enriquecimiento de la tarea y un acceso más fácil a la información. Las estrategias de gestión del trabajo, particularmente en algunos sectores más cualificados o para algunos grupos de profesionales, se han centrado en fomentar una organización del trabajo que permita la autonomía del trabajador, el enriquecimiento de la tarea, la ruptura de jerarquías, etc. En muchos casos, estas se han traducido en un mejor equilibrio entre la vida privada y la pública y una reapropiación y gestión del tiempo, lo que ha permitido, en algunos casos, una reducción del stress y una mejor salud psicológica (Duval y Jacot, 2000).

Sin embargo, el impacto de este proceso de recuperación de la soberanía del trabajador en el lugar de trabajo es relativo. Esta dotación de espacios de autonomía no es necesariamente sinónimo de una democratización en el seno de las empresas, ya que, como muestra Maroy (1997), es una autonomía profundamente delimitada y enmarcada que no se remonta a las decisiones estratégicas más importantes. Esta autonomía está al servicio de la empresa, y no es utilizable como estrategia de construcción de espacios al servicio de los trabajadores (potencialmente ilícitos y de oposición a aquélla), como era el caso en las organizaciones más tayloristas y rígidas del trabajo (Piray, 1997).

A su vez, importantes riesgos acompañan a estos desarrollos. El trabajo es cada vez más abstracto, hay una exigencia de actuar rápidamente, hay una continua demanda de calidad total, una dificultad de medir y, por tanto, de controlar y regular políticamente el tiempo de trabajo, ante una demanda de disponibilidad total. Estas formas de organización flexibles han ido así acompañadas de una tendencia a la intensificación del trabajo y a un aumento de la carga de trabajo. Las innovaciones tecnológicas son en parte instrumentos de esta intensificación del trabajo. Un ejemplo ilustrativo de estos desarrollos son los modos de gestión de los cuadros y técnicos profesionales (Bouffartigue y Bouteiller, 2000). Frente a las regulaciones colectivas del tiempo de trabajo, establecidas para el conjunto de trabajadores de una empresa o sector, que han permitido la reglamentación jurídica de la duración del trabajo, un número creciente de trabajadores permanecen en la actualidad al margen de la regula-

and the modernisation of its very structure. "Document for the presidency, Employment, Economic reforms and Social cohesion-towards a Europe based on innovation and knowledge». Council of the European Union, 12.1.2000, 5256/00, p. 14. 
ción del derecho del trabajo. Si el modo de evaluación y retribución del trabajo se ha basado fundamentalmente en el tiempo pasado en los locales del empleador, en el caso de este colectivo la evaluación de la prestación de trabajo se basa en los resultados llevados a cabo. Resultado de ello es que la referencia del tiempo de trabajo propia de la sociedad industrial bajo la que se ha desarrollado el derecho laboral ha dejado de ser pertinente, favoreciéndose lógicas de gestión más personalizadas. A cambio de una autonomía en el trabajo, los cuadros muestran su adhesión a los fines de la empresa y una disponibilidad incondicional. Plantean los autores cómo, en este caso, la remuneración depende de las competencias individuales para cumplir sus objetivos lo más rápidamente posible, más que el tiempo dedicado a ello. Resultado de esto es la prolongación indefinida de las jornadas laborales, la falta de control del tiempo de trabajo, la intensificación de las jornadas, etc. Un modelo de carrera que valoriza la disponibilidad creciente para la empresa, la individualización de la relación con el empleador, una remuneración por objetivos más que por la duración de la prestación, la obligación de resultados más que de medios (el tiempo pasado en la fábrica, por ejemplo), etc., explica la pérdida de control, por parte de este colectivo, del tiempo de trabajo.

Esta situación, en principio restringida a la «élite» de los trabajadores, se está extendiendo al conjunto de los trabajadores, avalada y legitimada por un discurso que hace de la flexibilidad un imperativo absoluto de la nueva economía. Frente a los ritmos sincronizados y a un tiempo de trabajo regulado, asistimos a la desregulación de la norma temporal de trabajo, de modo que ésta es ya incapaz de cumplir las funciones de evaluación y limitación de la prestación del trabajo, así como la sincronización y socialización de la vida de los trabajadores.

Por ello, si estas evoluciones han supuesto para algunos colectivos nuevas oportunidades, han ido también acompañadas de importantes riesgos. Como muestra un reciente informe de la European Foundation for the Improvement of Living and Working Conditions (2000), las condiciones de trabajo que acompañan a estos desarrollos están en cierto modo empeorándose. Aunque a veces se trabaje menos tiempo, se trabaja más rápidamente y con carácter más urgente y de modo más imprevisible (tiempos irregulares). Esto ha tenido importantes consecuencias en la carga mental de los trabajadores, un aumento del stress y una difusión de las fronteras entre el trabajo y el no-trabajo, etc. A las presiones de tiempo derivadas de la urgencia y de la necesidad de reaccionar rápidamente se une la apropiación de la vida privada, cuando los límites de tiempo y lugar de trabajo no están tan delimitados.

\section{Debilitamiento de la posición del trabajador frente al capital}

Estas nuevas formas de gestión de la mano de obra, que pueden ir desde el shared capitalism hasta el human resources management (flexible working 
methods, teamworking, etc.), han difuminado la ruptura de la clásica dicotomía entre el capital y el trabajo o el empleador y el empleado, minando identidades colectivas clásicas. Frente a la estructuración de la vida colectiva por las relaciones de clase propia de la sociedad industrial, basada en relaciones de producción y donde dominaba una representación de la sociedad en términos de conflictos de clase, el desarrollo de nuevas formas de gestión de los recursos humanos está promoviendo la difuminación de la histórica división entre el capital y el trabajo o entre el empleador y el empleado. Esto explica en parte la dificultad que expresan estos colectivos para transformar la indignación y la conciencia de injusticia en conflicto, estimulándose una perspectiva apolítica frente al problema.

Este proceso va acompañado de la desregulación jurídica del mercado de trabajo que está estimulando la diversificación de los tipos contractuales y relacionales con la empresa. De ahí que a la desreglamentación del mercado de trabajo estimulada por el discurso sobre las demandas de la nueva economía se una la individualización en el tipo de relaciones laborales.

\section{NTIC (Nuevas Tecnologias de la Información y de la Comunicación) y retaylorización del trabajo}

Si estas nuevas formas de organización del trabajo posibilitan en algunos casos el enriquecimiento de la tarea (variedad, mayor autonomía, aumento de las cualificaciones, descentralización, mayor capacidad de decisión) para los más cualificados, para otros implican la rutinización de tareas, la limitación del espacio dejado a la creatividad, la reducción de la autonomía, la descualificación. Supone la aparición de nuevas formas de taylorismo y la multiplicación de trabajos precarios, con horarios poco previsibles, fraccionados, irregulares y al margen de los ritmos sociales dominantes para los trabajadores menos cualificados (Bouffartigue y Bouteiller, 2000). Esta situación es tanto más importante cuando, tal y como muestra un estudio hecho acerca del impacto de formas de trabajo flexible ligadas a las tecnologías de la comunicación (Beaujolin, 1999), si en algunos sectores las competencias demandadas están vinculadas a trabajos con un contenido más enriquecido, que demanda la creatividad del trabajador, sin embargo, hay un número creciente de tareas cada vez más banales, que demandan únicamente aptitudes comportamentales (adaptación, aceptación de la flexibilidad, etc.).

Las NTIC, en muchos casos, más que fomentar la autonomía del trabajador, son instrumentos al servicio de una retaylorización de las tareas (véase el ejemplo de los call-centers; Duval y Jacot, 2000), con un control más estricto de la tarea, estimulándose nuevas formas de alienación del trabajo. Las nuevas tecnologías pueden también así servir para una vigilancia más sofisticada y una prescripción más estricta de la tarea. Gracias a las conexiones que engendran, se extiende el poder de control de las organizaciones sobre los individuos de 
una sociedad (Duval y Jacot, 2000). De ahí que estas nuevas tecnologías puedan reforzar las tendencias a la ampliación de la autonomía o servir para desarrollar nuevos controles y rigideces en la regulación del trabajo. A su vez, las NTIC pueden liberarnos del control estricto y la gestión del tiempo y espacio propio de las sociedades fordistas, pero también pueden crear nuevos sentidos del tiempo y espacio tanto o más alienantes que los precedentes.

\section{DE LA NOCIÓN DEL DESEMPLEO A LA DE FALTA DE EMPLEABILIDAD}

\section{Nuevos modelos de referencia de trabajador}

Un segundo eje en torno al que se articula este nuevo «contrato social», tal como suele presentarse en el discurso de las instituciones europeas, viene constituido por la noción de empleabilidad, noción ésta que contiene una teoría acerca de los factores de acceso al empleo. Este concepto estructura profundamente las explicaciones acerca de la exclusión social ${ }^{14}$. Frente a concepciones anteriores que entendían el desempleo como una falta de trabajo, lo novedoso de esta noción es el plantear la exclusión social en términos de falta de competencia personal. En este sentido, el discurso de la Comisión acerca de la sociedad basada en el conocimiento para todos hace de la empleabilidad el eje estructurante de la intervención frente a la exclusión social. La falta de empleabilidad es entendida como la carencia de las competencias personales necesarias para adaptarse a la economía basada en la información y conocimiento.

El factor competitivo, así, en este discurso de la «nueva economía», no serían tanto las cualificaciones como las competencias del trabajador ${ }^{15}$, lo que implica un cambio en las demandas sobre los perfiles profesionales. Nuevas exigencias normativas de polivalencia, autonomía y movilización del trabajador van a seguir a un modo de producción, estimulándose la redefinición del modelo normativo de lo que es un (buen) trabajador. Las competencias generales $^{16}$ (tales como la autonomía, la reactividad, la capacidad de formular diag-

14 "The knowledge based economy is increasingly challenging the adaptability of companies and the work force and setting new demarcation lines between the haves and the have nots in terms of skills, qualifications and competencies». "Communication from the Commission. Building an inclusive Europe». Brussels, 1.3.2000, COM (2000) 79 final.

${ }^{15}$ Se entiende por cualificaciones los conocimientos que una persona aprende a partir de una formación más o menos formalizada en instituciones educativas y acreditada con un diploma. Las competencias, por el contrario, son un componente de la actividad laboral más informal y más psicosocial; más que conocimientos precisos y técnicos, son orientaciones hacia el trabajo, actitudes hacia el cambio y disposiciones frente a la empresa (Alaluf y Stroobants, 1994; Zimmermann, 2000).

${ }^{16}$ More generally, Europe's population, and young people in particular, must have extensive access to new basic skills- they must be helped to develop a capacity to learn and to resolve problems; they need an appreciation of science and technological skills, they will need to be able to use information technologies, to develop a sense of initiative and entrepreneurship, and to be active, free and responsible citizens. "Communication from the Commission. Building an inclusive Europe». Brussels, 1.3.2000, COM (2000), p. 11. 
nósticos, etc.; Valenduc, 2000) van a pasar a ser el eje articulador de la valoración (de la empleabilidad) de los demandantes de trabajo. Frente a las anteriores exigencias sobre los perfiles profesionales ligados a la noción de cualificación (capacidad de realizar operaciones en una organización del trabajo en donde hay una parcelación de tareas y una separación entre la concepción y la ejecución del trabajo), los nuevos modelos profesionales demandan cualificaciones no vinculadas a tareas operatorias o a puestos de trabajo concretos, sino que son demandas más abstractas, vinculadas a funciones generales. En este contexto son particularmente relevantes los componentes no materiales de la actividad profesional (abstracción, reactividad, capacidad de gestión de la incertidumbre). Si la noción de cualificación se define por referencia a un proceso colectivo (las cualificaciones dependen de la organización del trabajo), la noción de competencia tiene un importante referente individual. Los usos de esta noción de competencia son así portadores de un tratamiento individualizado del trabajador (Zimmermann, 2000).

Por ello, esta noción de competencia, tal como la entendemos aquí, presenta dos facetas, una más psicológica (implicación y disponibilidad del trabajador) y otra más organizacional (formación continua). Por un lado, la implicación del trabajador en su trabajo es importante, ya que para hacer frente a una situación de cambio constante, de complejización social e interpersonal, de inestabilidad e incertidumbre, se requeriría no tanto las manos o la cabeza (disposición «mental» o «manual» del sujeto), sino su "alma», una disposición y entrega total. Por otro lado, dada la rápida obsolescencia de los conocimientos profesionales, de las fusiones de las empresas y las adaptaciones de nuevos útiles, se reclama a los trabajadores que mantengan una actitud constante de adaptación, actualización de conocimientos y de formación continua. Este entorno inestable sometería al individuo a una situación permanente de alerta. La formación permanente pasa a ser vista como un útil de supervivencia personal.

Los nuevos discursos del management están así construyendo un nuevo perfil de «buen» trabajador. Según éste, el trabajador, más que buscar el paternalismo y la sumisión que resultan de las relaciones estables, debiera dirigirse a alimentar un espíritu de iniciativa y progreso constante. La función de la empresa es así la de "ingeniería del alma» (Le Goff, 1999), en la que lo que une al trabajador con la empresa no es tanto la dependencia que se deriva de la seguridad en el empleo, sino la libertad que aporta la construcción de la empleabilidad del trabajador (otorgar los medios de su desarrollo personal). Implica así un nuevo "contrato moral" en el que la empresa otorga al trabajador los medios de desarrollarse y, a cambio, el trabajador acepta implicarse en la empresa. Las empresas pasarían así de ser lugares de sumisión a ser instituciones movilizadoras, lugares de aprendizaje e iniciativa.

A los trabajadores se les hace así responsables de su propia formación y de la gestión de su carrera. Esta gestión alimenta el "sufrimiento» (Dejours, 1998; Sennet, 2000) de los que tienen miedo de no responder a estas expectativas y presiones del trabajo, el miedo a la «in-competencia». 
Esta articulación en torno a la noción de "competencias» ha supuesto un importante cambio de paradigma en la concepción de los criterios de valoración del trabajador. No se trataría tanto de "conocimientos», "experiencia», etc., lo que explicaría, según esta perspectiva, el valor y la posición de los trabajadores en el mercado de trabajo, aspectos éstos objetivos y fácilmente medibles (y por tanto valorizables), sino de dimensiones subjetivas difíciles de aprehender y medir como la motivación, la actitud hacia el trabajo, etc. ${ }^{17}$, que dejan un gran margen de maniobra a la arbitrariedad por parte del empleador, perdiendo a su vez el trabajador el control de los criterios de valoración del trabajo.

Estos criterios de determinación del «buen trabajador» articulan, a su vez, una teoría acerca del carácter del desempleo, entendido en términos de falta de empleabilidad. Esta noción, articulada casi exclusivamente en torno a los factores de la mano de obra, plantea la responsabilización de los propios trabajadores frente a las dificultades de acceso o mantenimiento del trabajo. Haciendo de la carencia de "empleabilidad» el eje explicativo del problema, se rompe con concepciones anteriores del desempleo, basadas en la falta de trabajo, que impulsaron la «socialización de la responsabilidad» ${ }^{18}$. Con esta construcción de una teoría acerca del desempleo, se plantea también una tesis sobre cómo combatirlo. La cuestión social no sería así tanto la garantía de un trabajo como de

17 "Competencias comportamentales, definidas en términos tan generales, que todo el mundo es portador de ellas» (Alaluf y Stroobants, 1994).

${ }^{18}$ Diversos análisis históricos (Salais et al., 1986, y Topalov, 1994) muestran el papel que ha tenido la mutación social operada a finales del siglo XIX acerca de la concepción de la falta de trabajo en la institucionalización de un sistema de protección social dirigido a la defensa del trabajador frente a situaciones de riesgo temporal. Con la instauración de las sociedades industriales, no había una diferenciación clara entre el "pobre» y el "desempleado». A partir de la concepción individualista dominante, todos formaban parte de un grupo social de «indigentes», sujetos perezosos y poco motivados, responsables de su situación. La consideración política y social de la inactividad implicaba un diagnóstico moral. Una confluencia de condiciones va a cuestionar esta noción dominante de la carencia de empleo, como la movilización colectiva de la población, la emergencia de las relaciones colectivas de trabajo en la fábrica, la inadecuación de los medios represivos para hacer frente a los conflictos crecientes, etc. Ante la necesidad de control del desorden social que resultó de esta confluencia de factores, las instituciones de la época se dispusieron a la medición del problema, el desempleo, lo que facilitó el análisis de éste en función de sus causas. Se comienza así a diferenciar entre los factores individuales, pereza, forma de vida, enfermedad: «malos pobres», etc., y los sociales, pérdida involuntaria de trabajo: «buenos pobres». De este modo se va a generar la reformulación a nivel estadístico, jurídico y político del problema del desempleo. Este conjunto de factores explica el «nacimiento» del desempleo, esto es, la génesis de una concepción social con una entidad en sí misma, separada de la de "pobres o indigentes», en función del carácter personal (y voluntario) o social (e involuntario) de la situación. Se pasa así de un análisis de la exclusión en términos de "falta personal» a otra de «riesgo social». De este modo se planteó la necesidad de resolver socialmente un problema (responsabilidad y justicia social) que hasta ese momento había sido tratado en términos individuales y morales. La estrategia europea dirigida a los desempleados, formalizada con la noción de «empleabilidad», parece dirigirse justamente a la dirección contraria, favoreciéndose una lectura del desempleo en términos de "carencia o falta» (individual) más que de «riesgo» (social). 
la «empleabilidad", es decir, ofrecer al sujeto la oportunidad de formarse y actualizarse (Moss Kanter, 1999), dotándole de las así llamadas "competencias». Esta reconstrucción del problema va a tener importantes consecuencias en la distribución de responsabilidades sociales y, por tanto, en el papel reclamado socialmente a las instituciones sociales para su resolución.

Esta estrategia europea, articulada en torno al pilar de la empleabilidad, si bien no parece haber tenido grandes repercusiones en la disminución del desempleo, tal y como se desprende de diversos estudios evaluativos (Serrano, 2000), sí las ha tenido en la «invención del desempleo» (o de la falta de empleabilidad). Se hace pasar como falta de proyecto, de motivación, de formación, lo que no es sino falta de trabajo. Haciendo de los problemas sociales cuestiones personales (Crespo y Serrano, 2001), esta situación está actuando como mecanismo disuasivo que explica la dificultad para pensar y concebir el cambio y proyectar la confianza en el porvenir. Dada la autoculpabilización que se deriva de esta situación y la pérdida de legitimidad de una actitud combativa, esta situación interviene como mecanismo disuasorio de potenciales conflictos. Esta situación está favoreciendo, a su vez, estrategias individualizadas de salida de la situación, haciéndose de la adaptación a las nuevas demandas definidas por el capital la dimensión nuclear de evaluación de la empleabilidad de gran parte de los jóvenes. Este análisis, cuya explicación no aparece ligada a la organización social sino a faltas personales, permanece muy próximo a un diagnóstico moral.

\section{NUEVAS FUNCIONES DEL ESTADO SOCIAL: DE LA SEGURIDAD EN EL EMPLEO A LA ACTIVACIÓN DEL DESEMPLEADO}

\section{Refundamentación del sistema de prestación social}

La concepción de cohesión social planteada por las instituciones europeas con el discurso de la sociedad del conocimiento se articula no sólo en torno a un modelo de regulación social y de distribución de responsabilidades. Un tercer eje de este discurso es el modelo de sujeto ciudadano. Planteando la necesaria reforma y «modernización» de la Seguridad Social, se hace de la activación ${ }^{19}$ otro de los ejes en torno al cual se articula esta estrategia europea. La gestión anterior del desempleo, basada en la dotación de una subvención o seguro

19 Understanding the contemporary problems of the European social model and finding solutions to them requires starting from an adequate concept of welfare. Welfare is not only a guarantee of incomes in the face of social risks. Welfare is also based on personal services, quality of work and living opportunities. A positive strategy of renewal of the European social model needs to be adopted: aimed at raising employment levels and creating job opportunities for all; combining the principles of initiative, responsibility, social justice and solidarity. «Document for the presidency, Employment, Economic reforms and Social cohesion- towards a Europe based on innovation and knowledge», Council of the European Union, 12.1.2000, 5256/00, p. 14. 
frente a las situaciones de desempleo, es deslegitimada. Un doble argumento favorece esta posición discursiva, una crisis de financiación de la Seguridad Social y la así llamada "trampa del desempleo». La protección social no generaría, según esta interpretación, sino una relación de dependencia y subordinación frente al Estado social y es desincentivadora para salir de esta situación. Siguiendo una argumentación paralela al discurso clásico de la caña y el pez, la lógica sería más la de dar los útiles para salir de su situación de dependencia que la de (en)cubrirla.

Frente al discurso de las NTIC, que va acompañado de un tinte más tecnocrático, el discurso de la activación ${ }^{20}$ es un discurso moralizante. El consenso posterior a la segunda guerra mundial entre capital y trabajo, por el cual se garantizaba el crecimiento económico, el pleno empleo, la protección social y la solidaridad y estabilidad políticas, va a verse redefinido. El Estado social dejaría de ser visto como el organismo protector, para pasar a ser el sancionador y controlador de la «buena» conducta de sus ciudadanos. Su papel no consistiría tanto en la distribución de la riqueza como en la de oportunidades ${ }^{21}$, un Estado «animador» que recompensaría a los más dinámicos (Alaluf, 2000). Se instaura así un Estado de sospecha (contra la pasividad, la dependencia, el fraude). Así, los desempleados van a verse constantemente interpelados a fin de dar razón y muestra de su autonomía y responsabilidad. Esta forma de intervenir frente al desempleado acusado de "pasivo" (a menos que se demuestre lo contrario) se ha dirigido a la activación bajo un carácter autoritario.

La activación va a consistir así en una serie de dispositivos dirigidos a la «normalización» de la trayectoria profesional del desempleado. En un contexto de restricciones presupuestarias, se instalan medidas de vigilancia y detección de los buenos desempleados, entendiendo como tales a los sujetos que manifiestan una adhesión «moral» al ethos del trabajo y una actitud positiva frente a los cambios económicos. Este tipo de dispositivos ha acarreado diversos riesgos, como la génesis de un sector desprotegido del mercado de trabajo, el riesgo de dualización de la sociedad y la génesis de una nueva clase de "sirvientes». Con los recortes en la cobertura social del desempleo, se trata de fomentar una actitud de aceptación indiscriminada de cualquier trabajo. Uno de los objetivos de estos dispositivos sería así fomentar la disponibilidad, dando una prima

20 A pesar de las distintas formas de entender la activación en función de los contextos nacionales (Barbier, 2000), en gran parte de los países europeos hay una tendencia a reforzar el carácter punitivo de la activación.

${ }^{21}$ Thus, a comprehensive and co-ordinated policy approach to social inclusion should go for beyond the redistribution of prosperity by means of passive benefits paid to those left out by economic and social change- the challenge is not so much to increase redistribution but to manage our economies and societies in a way which maximises the potential for full participation, so as to reduce the waste of human resources and to achieve a fair distribution of opportunities... More than ever, encouraging and supporting a forward looking adaptation process that prepares all citizens for change offers the best prospect for the strengthening of solidarity and social cohesion. "Communication from the Commission. Building an inclusive Europe», Brussels, 1.3.2000, COM (2000), p. 8. 
a la buena voluntad. La justificación de esta presión se apoya en un argumento moral: hacer escapar al desempleado de la pasividad, no permitirle olvidar su obligación de contribuir a la sociedad. Ya que el modelo de individuo subyacente es el de un sujeto activo y emprendedor, el objetivo de estos programas sería desarrollar la creatividad y fomentar la capacidad de emprender el proyecto personal. Estos dispositivos parecerían así ir dirigidos a estimular una socialización moral del ejército de reserva. Sin embargo, este objetivo de disciplinamiento de un ejército de reserva dócil y preparado a aceptar cualquier trabajo, entra en aparente contradicción con el perfil de trabajador motivado e implicado que demanda la así llamada «nueva sociedad de la información».

Con nociones como la del workfare, se trata así de conciliar los dos polos de una falsa dicotomía entre welfare y work. Este discurso es particularmente peligroso pues ataca al Estado social de la forma más profunda, como si el principal enemigo del bienestar individual (que se ha identificado desde un discurso totalizador con la disposición de trabajo) fuera el Estado del bienestar.

\section{Nuevas concepciones de ciudadanía: el trabajo como deber civil}

Esta refundamentación del sistema de prestaciones sociales implica una nueva concepción de ciudadanía, no tanto una condición a la que todo sujeto por el hecho de nacer en un contexto le viene dada, sino como una conquista personal. Se introducen nuevas obligaciones y condiciones, que implican la reevaluación de los derechos y deberes inherentes a la condición de ciudadanía. Se pasa así de la noción de "necesidad», lógica que ha articulado las prestaciones sociales en las sociedades modernas, a la noción de "mérito», regla de derecho que regula el principio de reciprocidad (Morel, 2000). Estas políticas de workfare van dirigidas tanto a promover las obligaciones sociales de ciudadanía como a la reafirmación de una ética de trabajo. Implica la imposición de nuevos deberes donde antes no había sino derechos (Morel, 2000).

De este modo se estructura un nuevo tipo de fractura social, no tanto entre los sujetos favorecidos socialmente o no como entre los que manifiestan un deseo de insertarse y aquellos que no. Se establece así un sistema de sanciones que transforma la lógica de derecho (derecho a trabajar) en imposición de deberes o "contractualización de derechos», tan distinta de la noción universal de derechos de la persona.

Aparte de la dudosa evidencia del peso de la cobertura de desempleo en la desincentivación para la búsqueda de empleo (Bosco, 1997; Sinfield, 1997), se hace de la adopción de un trabajo un imperativo absoluto, como si el hecho de tener un trabajo, cualquier trabajo, fuera un fin en sí mismo. Estos dispositivos dirigidos a la activación y movilización del desempleado tienden a favorecer una adhesión incondicional de los trabajadores a la ética dominante del trabajo (motivación, esfuerzo, disciplina) y una revalorización de éste como bien último. Parecería como si los poderes públicos nacionales y locales, empobrecidos 
por la crisis económica, buscaran legitimar con una moral negativa el recurso a la protección social. Estos programas van a fomentar así una reorganización del principio de ayuda social basado en derechos sociales (y como tales universales), siendo sustituido por el principio del derecho discrecional o procedimental, según el cual se hace de una ética del trabajo el eje justificativo del derecho a recibir ayuda. Esta moralización de la ayuda social reactualiza una ética del trabajo superada por las transformaciones tecnológicas y el paro masivo. A pesar de la conciencia cada vez más extendida de la importancia de revisar una noción totalizadora y mercantilizada del trabajo, para la emancipación del individuo, este tipo de discursos basados en el workfare o en una socialización laboral del desempleado no hace sino reforzarla.

\section{REFERENCIAS BIBLIOGRÁFICAS}

Alaluf, M. (2000): Dictionnaire du prêt-à-penser, Charleroi: EVO.

Alaluf, M., y Stroobants, M. (1994): "Moviliza la competencia al obrero», Las competencias: el concepto y la realidad, CEDEFOP, 1, pp. 47-56.

Askenazy, P. (2000): "Le développement des pratiques "flexibles" de travail», en D. Cohen y M. Debonneuil (eds.), Nouvelle économie, París: La Documentation Française.

BARISI, G. (2000): «Economie de temps dans la net-economie et etapes nouvelles de soumission à la "pensée unique" du capitalisme neo-liberale», Issues, núms. 55-56, pp. 17-47.

Beaujolin, F. (1999): La gestion des compétences. Etudes de cas commentées, París: Nathan.

Bosco, A. (1997): «Unemployment benefit systems», en A. Bosco y M. Hutsebaut (eds.), Social protection in Europe. Facing up to changes and challenges, Brussels: European Trade Union Institute.

Bouffartigue, P., y Bouteiller, J. (1999): «Le temps de travail est- il controlable et négociable», contribución al Seminario sobre La négociation collective dans la construction de la norme sociale de l'emploi, organised by the network "Emploi, Travail, Relations Professionnelles et Société» and the Institut Syndical Européen.

BRÖDNER, P. (2000): "The future of work in a knowledge based economy», contribución al European Meeting About the Knowledge Society.

Capelli, P. (1999): The New Deal at Work: Managing the Market-Driven Workforce, Boston: Harvard Business School.

Cohen, D., y Debonneuil, M. (2000): Nouvelle économie, París: La Documentation Française.

Crespo, E., y Serrano, A. (2001): "The individualisation of labour, job insecurity and vulnerability: young people's experience of work in Spain", Transfer, 7 (2): 289-308.

De Coster, M. (1998): «Les divisions sociales du travail», en M. de Coster y F. Pichault (eds.), Tralté du Sociologie du Travail, Bruxelles: De Boeck.

Dejours, C. (1998): Souffrance en France. La banalisation de l'injustice sociale, París: Seuil.

Durand, J. P. (2000): "Flux tendu et modèle de la compétence: une révolution tranquille», Issues, núms. 55-56, pp. 47-71.

Duval, G., y Jacot, H. (2000): Le travail dans la société de l'information. Paradoxes et enjeux des Nouvelles Technologies d'Information et de Communication, París: Editions Liason.

European Foundation for the IMProvement of THE Living and Working Conditions: Press Release, 13.12.2000.

LE Goff, J. P. (1999): La barbarie douce. La modernisation aveugle des entreprises et de l'école, París: La Decouverte. 
MAROY, C. (1997): «Rapport à la norme et transformation des modes d'organisation de la production et du travail en entreprise», en J. De Munck y M. Verhoeken (eds.), Les mutations du rapport à la norme. Un changement dans la modernité?, De Boeck, pp. 107-120.

Morel, S. (2000): Les logiques de la reciprocité. Les transformations de la relation d'assistance aux Etats-Unis et en France, París: PUF.

Rubenson, K., y Schuetze, H. (eds.) (2000): Transition to the knowledge society. Policies and strategies for individual participation and learning, Columbia: Institute for European Studies.

SAlAis, R.; BAVEREZ y ReYNAUd (1986): L'invention du chômage. Histoire et transformation d'une catégorie en France des années 1890 aux années 1980, París: Presses Universitaires de France.

SENNET, R.: La corrosión del carácter, Barcelona: Anagrama.

Serrano Pascual, A. (ed.) (2000): Tackling youth unemployment in Europe, Brussels: ETUI.

Sinfield, A. (1997): «Blaming the benefit: the costs of the distintion between active and passive programmes", en A. BosCO y M. HuTSEBAUT, Social protection in Europe. Facing up to changes and challenges, Brussels: ETUI.

Topalov, C. (1994): La naissance du chômeur 1880-1910, París: Albin Michel.

Valenduc, G. (2000): "Vers une "société de la connaissance"?», Infose, núm. 6.

ZimmermanN, B. (2000): «Logiques de competences et dialogue social», contribución al Seminario Social Competences, Berlín, 6-7 enero.

\begin{abstract}
The discourse on the knowledge and information society has been integrated and popularised by European institutions and vinculated to the European social project. This discourse implies, among other things, a redefinition of social cohesion, that is characterised by a crisis context. This crisis is extended on three axis: a labour market regulation, an unemployment and a citizenship crises. The new social contract that the European Union institutions propose is articulated around these three axes: faced with the crisis of the market regulation, the discourse of work flexibility; faced with the crisis of unemployment, the discourse of employability and faced with the model of social intervention based on citizen's rights, the discourse of activation and personal responsibility.
\end{abstract}




\section{DEBATE}

